\title{
Das Brust-Zentrum Zürich Seefeld
}

\author{
Christoph Rageth \\ Brust-Zentrum Zürich Seefeld, Switzerland
}

\section{Vorgeschichte}

Als in den 1990er Jahren die minimal invasiven Methoden (stereotaktische und Ultraschall-gesteuerte Stanzbiopsien und Vakuumbiopsien) aufkamen und als immer mehr Artikel aufzeigten, dass die Brustkrebsbehandlung in spezialisierten Einheiten bereits nach 5 Jahren $\mathrm{zu}$ einer verminderten Brustkrebsmortalität führt, kam im Jahre 2000 der Gedanke auf, am öffentlichen Spital, wo der Autor damals als Chefarzt der Frauenklinik tätig war, ein Brustzentrum zu gründen. Dies hätte einen Raumbedarf (für die Stereotaxie-Einrichtung), eine apparative Aufrüstung und auch einen Kooperationsbedarf mit den umliegenden Spitälern bedingt. Die Kooperation wäre gut möglich gewesen, hingegen wurde die apparative und räumliche Aufrüstung zu schwierig, so dass die Realisierung eines solchen Zentrums auf privater Basis ins Auge gefasst wurde.

Bei der Raumsuche und der belegärztlichen Akkreditierung war die Privatklinik Hirslanden sehr behilflich, hingegen musste das 2 Mio. Euro umfassende Projekt durch private Unterstützung finanziert werden. Glücklicherweise fanden sich hochrangige schweizerische Experten (Prof. Rainer Otto, Radiologie und Prof. Hans-Jörg Senn, Onkologie), welche das Projekt von Anfang an tatkräftig unterstützten und eine Konsiliartätigkeit zusagten.

\section{Eröffnung des Brust-Zentrums}

Am 1. September 2001 wurde das Brust-Zentrum eröffnet, aber bereits in den 2 Wochen vor der eigentlichen Eröffnung hatte sich die Neuigkeit herumgesprochen, so dass schon im August 2001 zwischen den Umzugskisten Untersuchungen stattfanden und bereits die ersten Mammakarzinome an der Klinik Hirslanden operiert werden konnten. Seither wurde das Brust-Zentrum kontinuierlich ausgebaut und seit einigen Jahren suchen uns täglich etwa 20 neue Patientinnen auf. Die
Mehrheit der Patientinnen wird uns konsiliarisch zugewiesen, aber viele Frauen stoßen auch durch Bekannte oder das Internet auf uns.

\section{Personelles}

Das Kernteam setzt sich heute aus 6 Gynäkologen (4 davon sind auch operativ tätig), einem Chirurgen, 2 Radiologen, einer Studienärztin, einer Breast-Care-Nurse und einer Psychologin zusammen. Das Onkozentrum der Klinik im Park mit seinen 6 Onkologen betreibt konsiliarisch die Onkologiestation in unseren Räumen und an 2 Tagen pro Woche werden auch Chemotherapien auf 6 Therapieplätzen unter Leitung der medizinischen Onkologen durchgeführt. Einer der Onkologen (Prof. Gerd Nagel) hat sich auf die komplementärmedizinische Betreuung spezialisiert und eine Onkologin (Prof. Monica Castiglione) führt eine Zweitmeinungs-Sprechstunde an je einem Tag pro Woche. Die Radioonkologieabteilung der Klinik Hirslanden mit ihren 3 Radioonkologen betreut die meisten unserer Patientinnen und nimmt an der wöchentlichen Tumorkonferenz teil. Die Pathologie des Universitätsspitals Zürich führt die zytologischen und histologischen Analysen durch, betreibt seit November 2009 auch ein Schnellschnittlabor an der Privatklinik Hirslanden und nimmt ebenfalls an der wöchentlichen Tumorkonferenz teil. Während die onkoplastische Mammachirurgie (tumoradaptierte Reduktionsplastiken sowie sämtliche intramammäre Rekonstruktionstechniken) durch das Kernteam des Brust-Zentrums durchgeführt werden, führen die 3 plastisch-chirurgischen Konsiliarärzte die übrigen Eingriffe (Primär- und Sekundärrekonstruktionen mittels Eigengewebe- oder Implantatrekonstruktion) durch. Die genetischen Beratungen werden durch ein Teammitglied des Tumor- und Brustzentrums (ZeTuP) in St. Gallen (Dr. Barbara Bolliger) in unseren Räumen wahrgenommen. Die übrigen 20 Angestellten verteilen sich wie folgt: Leitendes Personal und IT: 3, Sekretariat: 4, MTRA: 4, MPA: 6, Study-Nurses und Datamanagerinnen: 3. Die Physiotherapie findet in externen Instituten statt.

\section{KARGER}

Fax +497614520714

Information@Karger.de

www.karger.com (c) 2010 S. Karger GmbH, Freiburg

Accessible online at:

www.karger.com/brc
PD Dr. med. Christoph Rageth

Brust-Zentrum

Seefeldstr 214, 8008 Zürich, Switzerland

Tel. +41 44380 76-60, Fax -61

c.rageth@brust-zentrum.ch

www brust-zentrum.ch 


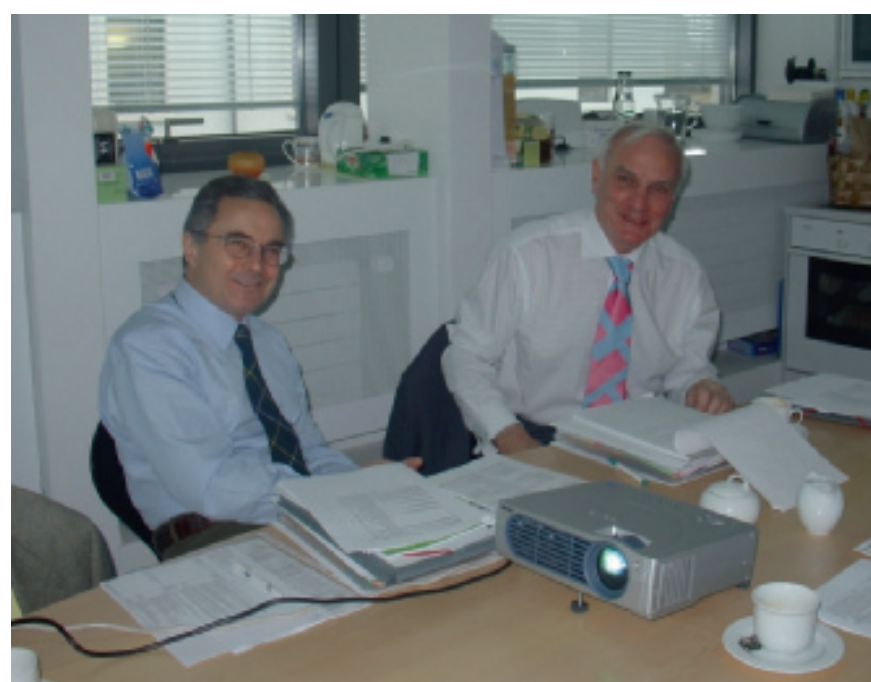

Abb. 1. Luigi Cataliotti (links) und Roger Blamey am 16.12.2003 im Brust-Zentrum Zürich Seefeld anlässlich der Initiierungsvisite.

\section{Räumliche und apparative Entwicklung}

Die Startfläche betrug $180 \mathrm{~m}^{2}$ und konnte sukzessive auf die heutige Betriebsfläche von $1000 \mathrm{~m}^{2}$ ausgebaut werden. Im Jahre 2007 wurde die digitale Mammografie (CR-System) eingeführt und im vergangenen Jahr wurde dieses Gerät durch zwei volldigitale Mammografie-Systeme ersetzt. Zusätzlich werden 8 Ultraschallplätze betrieben. 2-3-mal pro Monat führen wir mit Hilfe eines spezialisierten Teams (Alliance Medical GmbH, Castrop-Rauxel, Deutschland) Stereotaxien durch. Operationen finden an der Privatklinik Hirslanden statt und für die Patientinnen ohne Zusatzversicherung haben wir dank guter Kooperation mit einem öffentlichen Spital (Zollikerberg) eine begrenzte Anzahl Betten zur Verfügung. Ende 2007 schaffte die Klinik Hirslanden gemeinsam mit der Universitätsklinik den Intrabeam ${ }^{\circledR}$ (Carl Zeiss Meditec $\mathrm{GmbH}$, Jena, Deutschland) ein Gerät zur intraoperativen Radiotherapie, an und seither konnten gut 120 Patientinnen mit dieser Methode behandelt werden; 13 davon in der TARGIT Studie (intraoperative vs. postoperative Radiotherapie).

\section{Zertifizierung}

Auf Anregung des damaligen Ordinarius für Gynäkologie und Geburtshilfe an der Universitätsfrauenklinik Zürich (Prof. Urs Haller) begannen wir im Jahre 2002 eine Kooperation und bemühten uns als erste Einheit um eine Zertifizierung durch die EUSOMA. Abbildung 1 zeigt die erste durch die EUSOMA durchgeführte Initiierungsvisite am 16. Dezember 2003. Die Zertifizierung fand schließlich im Frühjahr 2006 statt. Mittlerweile könnten sowohl das Universitätsspital wie auch das Brust-Zentrum Seefeld die EUSOMA-Bedingungen selbständig erreichen, aber die Eingabe zur Rezertifizierung haben wir erneut gemeinsam durchgeführt.

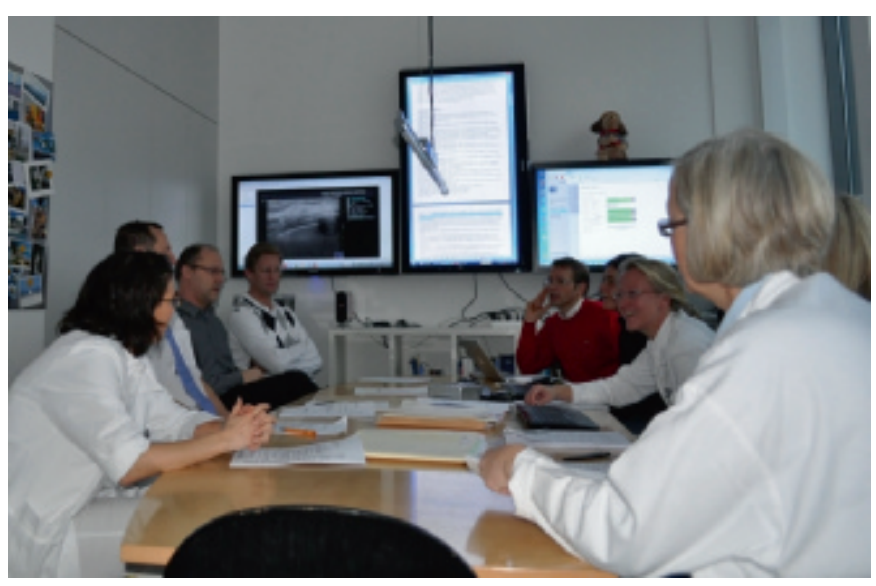

Abb. 2. Wöchentliche Diagnostikkonferenz (Vorstellung aller präoperativen Fälle und der diagnostischen Problemfälle). Auf hochauflösenden Monitoren werden unter anderem Ultraschallbilder und Mammografien präsentiert.

Ärztenetzwerk, Diagnostikkonferenz und Tumorboard

Der Erfolg des Brust-Zentrums Seefeld beruht in erster Linie auf der Kooperation mit den Zuweisern und der vom BrustZentrum angebotenen Fortbildungstätigkeit sowie der Tatsache, dass das gesamte Team sich fast ausschließlich der Brustdiagnostik und -therapie widmet. Für gynäkologische Patientinnen besteht seit 2003 ein Aufnahmestopp und seit 2004 führen wir selbst keine gynäkologischen Eingriffe mehr durch. Wir erachten es für sehr wichtig, dass die Patientinnen soweit wie möglich in ihrer hausärztlichen bzw. eigenen gynäkologischen Betreuung verbleiben können. Die Operationen werden sehr oft zusammen mit operativ tätigen Zuweisern durchgeführt - sei es, dass diese selbst operieren, oder assistieren. Hierbei wird auch auf die Einhaltung der Zertifizierungskriterien geachtet, welche insbesondere die prä- und postoperative Fallbesprechung und die Präsenz eines chirurgisch tätigen Kernteam-Mitglieds bei einer Operation beinhalten. Dies hat zu einer gewissen Selektion der Zuweiser geführt, indem heute jene Zuweiser, welche keine präoperative Besprechung und keine operative Zusammenarbeit wünschen, ihre Patientinnen für Mammografien und Punktionen eher an andere Stellen überweisen. Die Netzwerkärzte können ihre Patientinnen zur präoperativen Besprechung auf elektronischem Weg unserer wöchentlichen Diagnostikkonferenz (mit jeweils zirka 15 Fällen) anmelden (http://www.brustzentrum.ch/aerztenetzwerk/anmeldung_diagnostikkonferenz2. htm) und auch daran teilnehmen. Dabei werden auf hoch auflösenden Bildschirmen die Sonografien und Mammografien präsentiert (Abb. 2) und es werden auch alle hausinternen operativen Fälle (benigne und maligne) präoperativ sowie die diagnostischen Problemfälle im Team diskutiert. Jährlich findet eine Fortbildung, das Senologie-Update statt, an welcher jeweils zwischen 300 und 400 Ärztinnen und Ärzte teilnehmen. 


\section{Statistik}

Vom 1.9.2001 bis zum 31.12.2009 suchten 29000 Patientinnen das Brust-Zentrum auf. Die Kennzahlen für 2009:

16500 Konsultationen. 8200 Mammografien. Mammasonografien: 8960 bei 7282 Patientinnen. 1942 Punktionen, davon 69\% Stanzbiopsien, 25\% Feinnadelpunktionen, 4\% kombiniert und 2\% Mamillensekret-Zytologien. 8\% der Zytologien waren maligne, während 307 (21\%) der Stanzen ein invasives Karzinom und weitere 58 (4\%) eine Risikoläsion ( $\mathrm{ADH}$, FEA, Papillom, Phylloidtumor, LN) oder ein DCIS zeigten. Die 517 Vakuumbiopsien waren vorwiegend stereotaktisch (56,5\%) und sonografisch gesteuert (40\%), während die MRgesteuerten (durchgeführt am Institut für Radiologie der Klinik Hirslanden) 3,5\% ( $\mathrm{n}=18)$ ausmachten. Neue Patientinnen mit Mammakarzinom: 450, 317 davon durch das BrustZentrum operiert (234 in der Klinik Hirslanden, 6 auswärts und $77 \mathrm{im}$ Zollikerberg). Seit Aufnahme der Studientätigkeit vor gut 2 Jahren konnten bisher 81 Patientinnen in Studien eingeschlossen werden: ALTTO 12, Beatrice 1, CARIATIDE 6, NEOSPHERE 3, SENTINEL-IBCSG 23 12, SOFT-IBCSG 24 5, SOLE-IBCSG 35 16, TARGIT 26.

\section{Zukunft}

\section{Ärztenetzwerk}

Im laufenden Jahr wird wohl die Zertifizierung von Brustzentren durch die Schweizerische Gesellschaft für Senologie zusammen mit der Schweizerischen Krebsliga beginnen. Ein Kernpunkt dabei wird die Bildung von Ärztenetzwerken sein, weshalb sich das Brust-Zentrum bereits intensiv darauf vorbereitet hat. Es fanden Treffen mit interessierten Ärztinnen und Ärzten statt, um die zukünftige enge Kooperation im Hinblick auf die Label-Vergabe «Mitglied des Ärztenetzwer- kes Brust-Zentrum Zürich Seefeld» zu koordinieren. Offen ist noch, in wieweit Videokonferenzsysteme für die prä- und postoperativen Fallbesprechungen notwendig sind. Das Kooperationssystem soll so ausgelegt und verfeinert werden, dass im Normalfall eine Patientin nicht im Brust-Zentrum erscheinen muss. Die Koordination der Tumornachsorge und die Datendokumentation im Hinblick auf die Qualitätssicherung und die Benchmarks würden dann jedoch durch das Brust-Zentrum wahrgenommen.

\section{Brustklinik}

Des Weiteren wäre natürlich auch die Integrierung der stationären Behandlung in unsere Räume anzustreben. Dies ist aber vorläufig politisch nicht realisierbar, wenn auch Patientinnen ohne Zusatzversicherung ihre stationäre Behandlung in der Brustklinik haben sollen. Hierzu bräuchte es einen Leistungsauftrag, was nach einer Anfrage bei der kantonalen Zürcherischen Gesundheitsdirektion zurzeit illusorisch erscheint.

\section{Ausbildungsfunktion}

Im Brust-Zentrum finden Studentenkurse statt und es werden auch mehrere Dissertanten betreut. Bei den derzeitigen Fallzahlen müsste eigentlich durch das Brust-Zentrum auch eine Ausbildungsfunktion wahrgenommen werden. Bisher haben wir dies jedoch nicht geplant, weil dann die individuelle Betreuung der Patientinnen leiden würde. Als private Institution haben wir bereits jetzt beim derzeitigen Arbeitsanfall Probleme, nicht als «Fabrikbetrieb» wahrgenommen zu werden. Deshalb planen wir eher die Ausweitung der Kooperation mit öffentlichen Kliniken, um auch für die nicht zusatzversicherten, durch unser Institut abgeklärten Mammakarzinompatientinnen die Kontinuität der Behandlung aufrecht erhalten zu können. 